Bulletin de la Société Royale des Sciences de Liège, Vol. 85, 2016, p. 409 - 417

\title{
Pervasive and Ubiquitous Mobile-health application for pregnancy's mother on android smart phone (Non-Stress Test application)
}

\author{
KOZOUIE Nasim ${ }^{1}$, FOTOHI-GHAZVINI Faranak ${ }^{2}$, MINAIE-BIDGOLI Behrouz ${ }^{3}$ \\ ${ }^{1}$ Qom University, Iran \\ N_khozooyi2003@yahoo.com \\ 2. Qom University, Iran \\ f-fotouhi@qom.ac.ir \\ ${ }^{3}$ Iran University of Science and Technology, Iran \\ b_minaei@iust.ac.ir
}

\begin{abstract}
The emerging m-Health concept represents the evolution of e-health systems for healthcare using Smartphone applications with self-monitoring and sensing capabilities can help in disease prevention and health interventions. Disease risk conditions can be mapped to data collected via phone sensors and self-reports, providing users and doctor's access to rich health data or broadcasting alerts to caregivers. Non-Stress Test is a most important for the pregnant mothers with abortion risk. It is frequently carried on when they are faced with the abortion risk over 28 weeks of gestation. In this paper, we present a mobile healthcare application for pregnant mother as Non-Stress Test without relying on the Internet connection.
\end{abstract}

Keywords: mobile healthcare applications, Non-Stress Test, FECG, m-health, pregnancy, and abortion risk.

\section{Introduction}

\subsection{M-Health}

Development of mobile technology has enabled a new opportunity to enhance health care. mHealth has emerged has an extension of e-Health, which involves the use of mobile devices such as smart phones and smart watches to help patients who may need remote monitoring, share information with health systems, etc. [9]. Some of the features of these devices include third and fourth generation mobile telecommunications, global positioning system (GPS), Bluetooth technology, Wi-Fi, voice recognition, fingerprint scanners, among others advanced sensors that make a mobile device a potential tool.

In order to propose a health solution it is necessary to take into account the challenges related to mobile health domain [10]:

- Reliability: Health applications on a user mobile device concern about how much, hardware, sensors and software components can be trust and how ensure the integrity of the data.

- Customization \& Adaptability: Due to the diversity of problem situations, a m-Health solution should be able to be adjustable according to the user's needs.

- Security and privacy: The protection of health data is a big challenge. Insecure data storage could be a vulnerary ability for the user's privacy. 
- Resource limitations: Hand held devices have limited resources, some of the features like advanced sensors might need too much energy or are just too expensive to be implemented.

- Usability: Each user of m-Health technology might have different needs, so it is hard to create an easy to use graphical interface for everyone. Physical constraints like small screen can affect usability of the applications.

- Efficient data handling: Accumulated sensors are able to collect a big amount of data, but it is not necessary to store every. Additionally, techniques to analyze and retrieve data have to be included [11].

\subsection{Foetal Non-Stress test}

The Foetal Non-Stress test (NST) is a simple, non-invasive test performed in pregnancies over 28 weeks gestation. The test is named "non-stress" because no stress is placed on the foetus in during the test.

1.2.1 How is a NST Performed?

The test involves attaching one belt to the mother's abdomen to measure foetal heart rate and another belt to measure contractions. Movement, heart rate and "reactivity" of heart rate to the movement are measured for 20-30 minutes. If the baby does not move, it does not necessarily indicate there is a problem; the baby could just be asleep.

The primary goal of the test is to measure the heart rate of the foetus in response to its own movements. Healthy babies will respond with an increased heart rate during times of movement, and the heart rate will decrease at rest. The concept behind a non-stress test is that adequate oxygen is required for the foetal activity and the heart rate to be within the normal range. When oxygen levels are low, the foetus may not respond normally. Low oxygen levels can often indicate a problem with the placenta or the umbilical cord.

\subsubsection{NST evaluation criteria are:}

A. The foetal movements: two moves for 20-30 minutes.

B. Foetal heart rate:

C.

Table 1.Risk Assessment of Foetal Heart Rate

\begin{tabular}{cc}
\hline Diagnose & Condition \\
\hline \hline Normal & 120 to 160 Beats per minute \\
Mild bracardia & 100 to 120 Beats per minute \\
Sever bracardia & Below 80 \\
Mild Tachycardia & 160 to 180 Beats per minute \\
Sever Tachycardia & More than 180 Beats per \\
& minute
\end{tabular}

A reactive non-stress result indicates that blood flow (and oxygen) to the foetus is adequate. A nonreactive non-stress result requires additional testing to determine whether the result is truly 
Bulletin de la Société Royale des Sciences de Liège, Vol. 85, 2016, p. 409 - 417

due to the poor oxygenation, or whether there are other reasons for foetal non-reactivity (i.e. sleep patterns, certain maternal prescription or non-prescription drugs).

\section{Related work}

Parents to be are downloading pregnancy apps for their Google Android and Apple iOS devices at increasing rates. Gone are the days when expectant parents needed to reference books or keep notebooks to find pregnancy information and track pregnancy details. Smart-phones allow us to have almost everything we need at our fingertips. ABC News reported in March 2013 that 47 percent of total mobile subscribers that use one or more health app use a pregnancy-related app, according to Byte Mobile's first quarter 2013 mobile analytics report. In below, it has given several pregnancies' mobile application.

\subsection{Fertility Friend Mobile}

For women who are trying to conceive, Fertility Friend Mobile is an extremely helpful and convenient companion. We found that getting to know this app required more time than some of the others, but that's because it is comprehensive and absolutely serious about helping you monitor your fertility. FertilityFriend.com started as an online fertility-charting pioneer in 1998, and has grown into a leader in terms of popularity and accuracy. The app, at face value, is simply a chart and calendar to track your period, fertility and ovulation days. Yet what goes into the tracking is a thorough account of your primary fertility signs including cervical fluid, menstruation, sexual activity and basal body temperature, it also accounts for secondary signs such as cervix position, texture and opening; any tests you've used; medications; physical specifics and situations such as bloating, backaches, exercise, drinking, etc.; mood; weight; and a custom field for anything else you'd like to note. You may set an alarm as a data-entry reminder.

\subsection{Kindara Fertility}

Whether you are pregnant, trying to avoid pregnancy or just trying to become better connected to your body's cycles, the Kindara Fertility app is worth downloading to your iPhone or iPad-it will be available for Android soon. We really liked its intuitive design, simplicity, soft color scheme, and helpful tutorial. This app charts your cervical fluid, basal body temperature, sexual activity and menstruation to help determine peak fertility.

\subsection{My Pregnancy Today}

My Pregnancy Today is Google Play's top pregnancy app. Moms and dads to be can track the pregnancy's progress day by day. In addition to information on how the mother's body is changing, features include foetal development images, a checklist for tracking things like appointments, videos, a due date calculator and a nutrition guide with recipes.

\subsection{Pregnancy Sprout}

American Baby Magazine and Med ART Studios developed this app, which combines brains and beauty. In other words, it achieves what few apps set out to: being both functional and pleasant to look at. While it is fun to use for tracking your pregnancy's progress and learning what is happening to your body - or your partner's body — as well as the baby developing within, it is also extremely helpful for staying organized. You can track doctor visits, access checklists for newborn essentials and what to pack for the hospital, and create your own to-do lists. As we have shown, there are many interesting approaches to monitor pregnancy's mothers. In the following sections we will describe our approach that aims to complete some of the existent features and to include new functionalities. The proposed mobile application helps to mother with abortion Risk. This application help theme to check foetal and reduce referred to the hospital. At this 
application, we have implemented Non-stress Test, NST is a non-invasive test that poses no known risks or side effects to mother or foetal. When there are abortion Risk after 28 weeks, this test is necessary.

\section{System description}

\section{Technologies used in the system}

\subsection{Back-end technology:}

With the increasing use of mobile devices, it is common that applications offer features such as security storage backups, data synchronization, and data exchange among others. Building independent applications that only have functions and save data in a mobile device is not always feasible. A back-end server component normally is needed to efficiently store and manage data of mobile components [15]. A mobile application that shares data with other mobile clients requires different architectural components such as Push notifications, web services, centralized data storage, etc.

\subsection{Back-end components:}

Require a scalable infrastructure to support high volume of users. There are several platforms that offer ready-made back-ends that can be integrated into new mobile applications. These ready-made back-ends are Called Back-end as a Service or simply BaaS. For instance, Parse [15] is a cloud BaaS that allows the development of applications that require a back-end with minimal effort. The main characteristics that Parse offers are the following:

i. Cloud data model: Creation of NoSQL tables to efficiently insert modify and search via an API.

ii. Push notifications: Sending of push notifications to users (previous user acceptance).

iii. Cloud code: Ability to run code on the server, which is useful for running jobs and automatic security validations after data changes.

\subsection{Eclipse ADT:}

ADT (Android Developer Tools) is a plug-in that provides a set of tools that can be integrated to the Eclipse IDE. It offers access to many features that help develop efficient mobile applications in the Android platform [16].

The main Eclipse ADT features are the following [16]:

i. Creation, building, packing, installation and debugging of An application

ii. Android SDK integration in the Eclipse IDE

iii. JAVA programming language environment and XML editor

iv. Integrated documentation of the Android API. 


\section{System design and implementation}

System hardware comprises of a microcontroller based ECG and accelerometers. Most mobile applications use 3 ECG electrodes, but for Foetal ECG (FECG) detection 1 electrode is enough. The proposed circuit is shown in fig.1 combined with accelerometer sensors. Three ECG electrodes have been used. The signal has been pre-processed, and sent to the mobile phone (Android OS). The FECG extraction has been carried out on the mobile phone. The peak detection algorithm (for Heart Rate) has been used.

\begin{tabular}{|c|c|c|c|c|c|}
\hline $\begin{array}{c}\text { ECG } \\
\text { elecrode }\end{array}$ & $\begin{array}{l}\text { ECG front } \\
\text { and } \\
\text { Amplifier }\end{array}$ & $\begin{array}{c}\text { Signal } \\
\text { filtering }\end{array}$ & $\begin{array}{c}\text { EKF } \\
\text { filtering }\end{array}$ & $\begin{array}{c}\text { Peak } \\
\text { detection }\end{array}$ & $\begin{array}{l}\text { Heart } \\
\text { Rare }\end{array}$ \\
\hline
\end{tabular}

Fig. 1: System Block Diagram for Foetal Heart Rate.

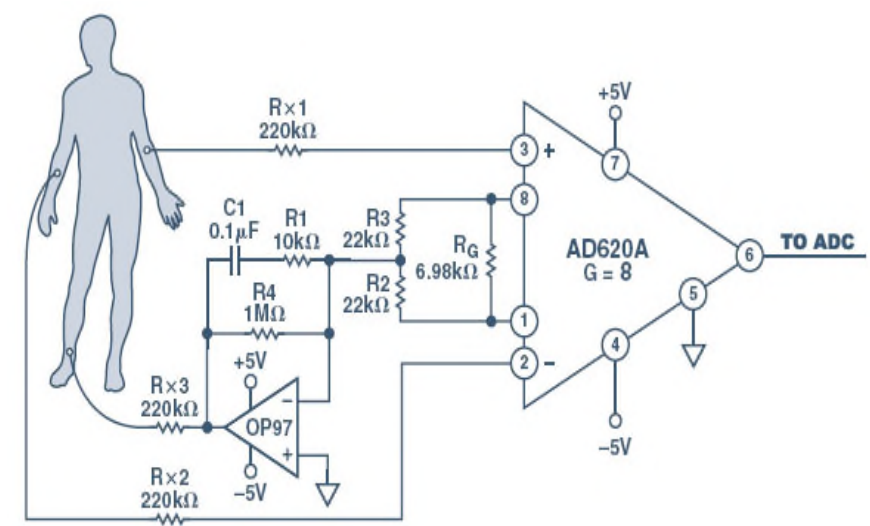

Fig. 2: ECG circuit

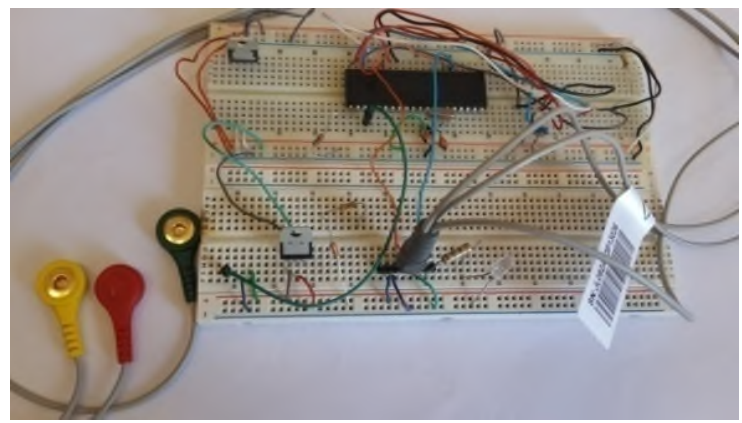

Fig. 3: System Hardware Implementation (ECG sensor)

\subsection{The FECG Extraction and the Foetal Heart Rate Measurement:}

The framework is based upon an extended Kalman Filter (EKF) for extracting the FECG from a single abdominal channel. The channel is described using a training database of 20 sample at one minute (maternal-foetal mixtures). The data was generated using the simulator, FECGsyn, used to generate a subset of the signals of the Physionet Challenge 2013. A single pass of the 
EKF (EKFS) was performed to cancel out the maternal ECG (MECG) in order to build an average FECG morphology. A dual EKF (EKFD, i.e. where both the MECG and FECG cycle morphology were modelled) was then applied to separate the three sources present in the signal mixture (noise, MECG and FECG). Normalized root mean square error and absolute QT error were calculated after EKFS and EKFD were extracted [for more detail refers to7].

Mat lab was used for implementing the EKFD filter and the peak detection algorithm. Then it was recorded to fit Android operating system on mobile phone. Then the heart rate could be calculated.

Count the number of QRS complexes over a 6 second interval. Multiply by 10 to determine heart rate. This method works well for both regular and irregular rhythms. If we can count $7 \mathrm{QRS}$ complexes, so the heart rate is 70 .

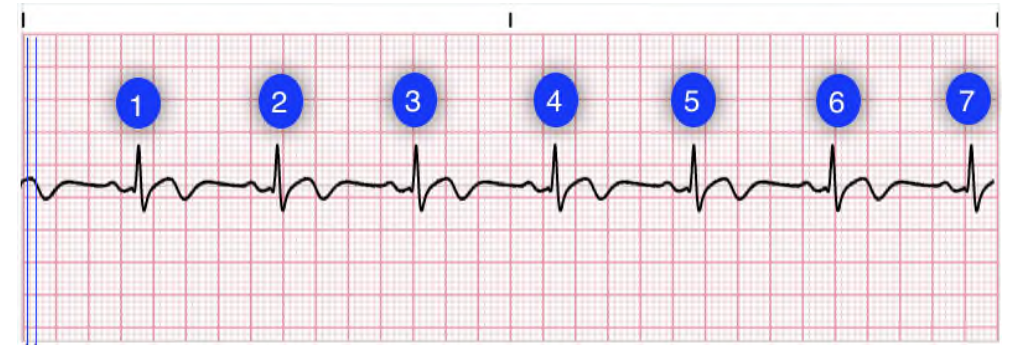

Fig 4: Calculating Heart Rate

\subsection{Movement detection:}

This process is done by the accelerometer sensors. The sensors were fitted on a belt that have been placed on the mother's abdomen. Severe changes in each of the axis $\mathrm{x}, \mathrm{y}, \mathrm{z}$ indicated that the foetus was moving. 
Bulletin de la Société Royale des Sciences de Liège, Vol. 85, 2016, p. 409 - 417

Table 2: Code for accelerometer sensor on Android

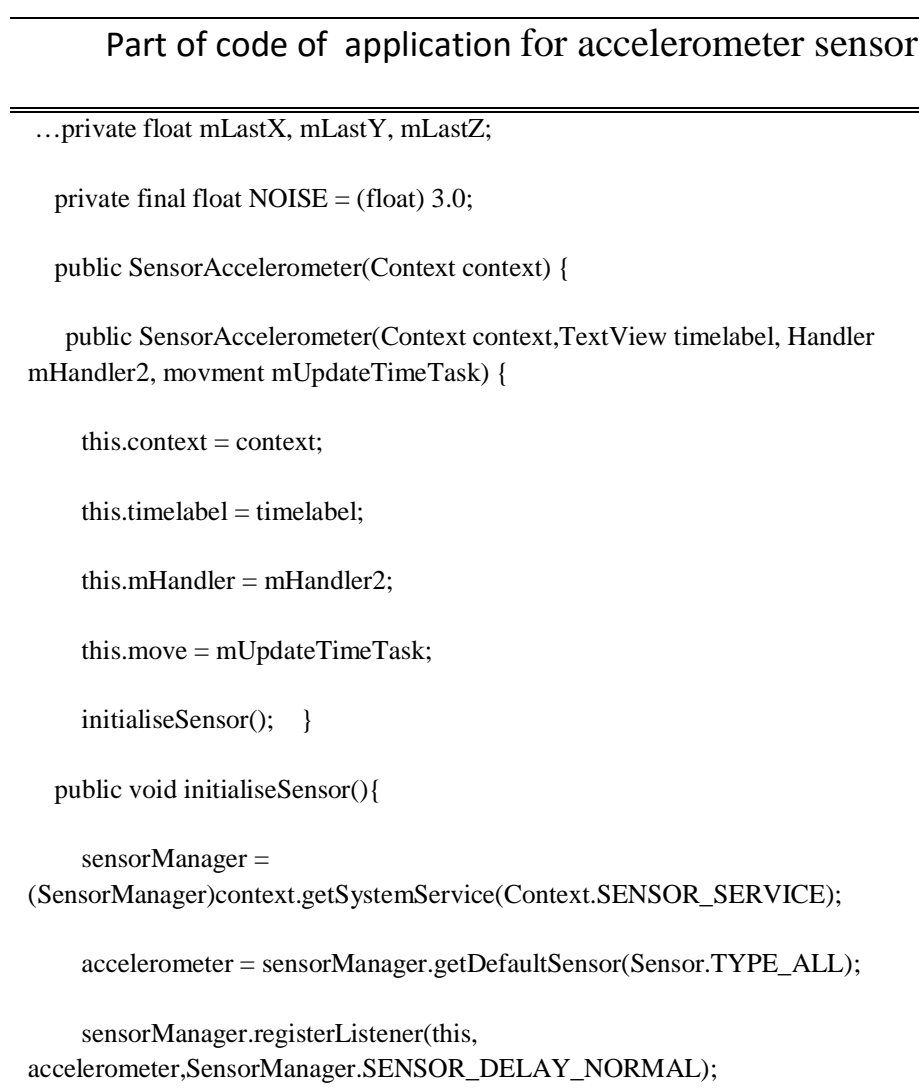

However, the experiment faced a challenge. If the mother was strongly laughing, sneezing or coughing there was a significant change in the accelerometer data. To overcome these challenges, SVM [14] method was used in data processing on the mobile phone.

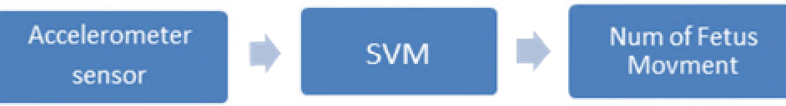

Fig 5: System Block Diagram for Foetal movment detection 
Bulletin de la Société Royale des Sciences de Liège, Vol. 85, 2016, p. 409 - 417

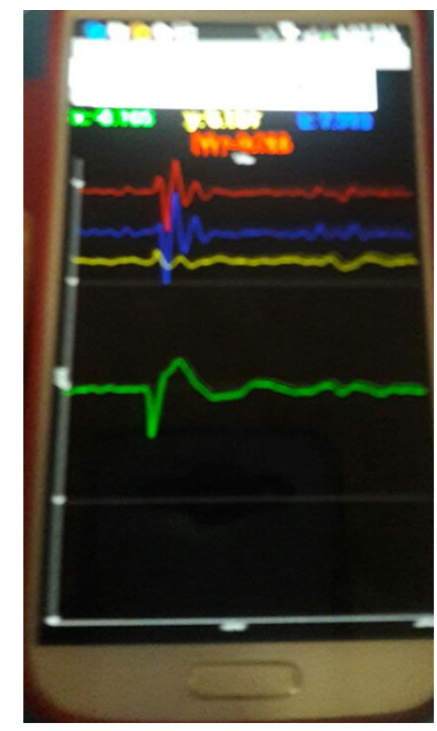

Fig 6: Foetal movement detection on Smartphone (Samsung) by embedded three-axis accelerometer sensor.

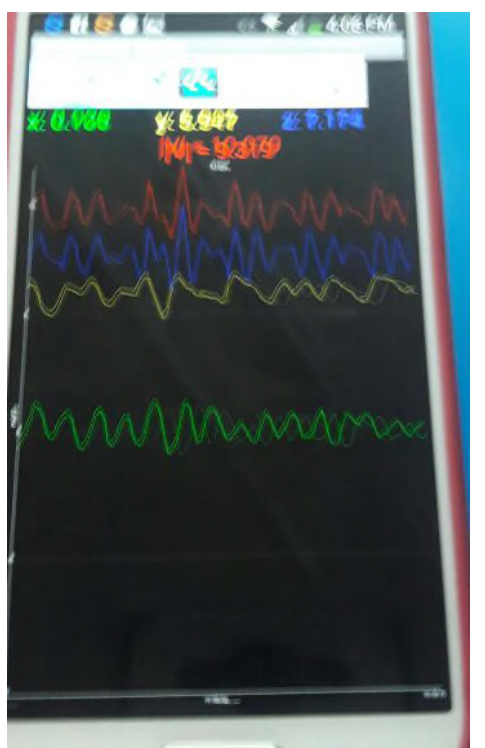

Fig 7: Other vibrations, such as laughing, coughing, etc... on Smartphone.

\section{Discussion and conclusions}

In this research we have proposed a mobile Non stress Test framework for pregnancy care. This framework belongs to M-healthcare applications. With this system, pregnant women could check her foetal's health at any place or any time without relying on Internet.

NST is a non-invasive test that poses no known risks or side effects to mother or foetal [12]. A reactive non-stress result indicates that blood flow (and oxygen) to the foetus is adequate. But we must know a nonreactive non-stress result requires additional testing to determine whether the 
Bulletin de la Société Royale des Sciences de Liège, Vol. 85, 2016, p. 409 - 417

result is truly due to the poor oxygenation, or whether there are other reasons for foetal nonreactivity (i.e. sleep patterns, certain maternal prescription or non prescription drugs).

But the way, when abnormal condition occurs, request check will be sent to the carer.

The application enables real-time monitoring and thus ensures that mothers are within a safe limit predefined by the caregivers, which makes it pervasive and ubiquitous.

\section{References}

[1] P.J. Marron, S. Karnouskos, D. Minder, A. Ollero, Applications, in: P.J. Marron, S. Karnouskos, D. Minder, A. Ollero (Eds.), The Emerging Domain of Cooperating Objects, Springer, Berlin, Heidelberg, 2011, pp. 125-176.

[2] J. Yick, B. Mukherjee, D. Ghosal, Wireless sensor network survey, Compute. Networks 52 (12) (2008) 2292-2330, http://dx.doi.org/10.1016/j.comnet, 2008.04.002.

[3] A. Pantelopoulos, N.G. Bourbakis, A survey on wearable sensor-based systems for health monitoring and prognosis, Trans. Syst. Man Cyber. 40 (2010) 1-12, http://dx.doi.org/10.1109/TSMCC.2009.2032660

[4] M. Domingo, context-aware service architecture for the integration of body sensor networks and social networks through the IP multimedia subsystem,

IEEE Commun. Mag. 49 (1) (2011) 102-108, http://dx.doi.org/10.1109/MCOM.2011.5681022.

[5]SANDEEP K. S. GUPTA et al, "Body Area Networks Safety, Security, and Sustainability", www.cambridge.org/9781107021020(e-book)2013

[6] Joachim Behar, Fernando Andreotti, Julien Osterl, Gari D. Clifford, A Bayesian Filtering Framework for Accurate Extracting of the Non-Invasive FECG Morphology, Computing in Cardiology Conference (CinC), IEEE2014

[7]Clifford GO, Sameni R, Ward J, Robinson J, Wolfuerg AJ. Clinically accurate foetal ECG parameters acquired frommaternal abdominal sensors. Am J Obstet Gynecol 2011; 205(1):47el-e5.

[8] Mobi Thinking, Global mobile statistics 2013 Part A: Mobile subscribers; handset market share; mobile operators, August 2013, http://mobithinking.com/mobile-marketing-tools/latest-mobilestats/a\#subscribers

[9] H. Chen and X. Jia, "New requirements and trends of mHealth," 2012, IEEE 14th Int. Conf. e-Health Networking, Appl. Serv. Heal. 2012, pp.27-31, 2012.

[10] A. Siddiqui, O. Koch, A. Rabie, and U. Handmann, "Personalized and Adaptable mHealth Architecture," 2014.

[11] Jeisson A. Vergara, Yesika M. Ram'irez, "A pervasive and ubiquitous mobile health application for tracking people with disabilities", 978-1-4673-9464-2/15/\$31.00 (C2015 IEEE

[12] http://www.webmd.com/baby/nonstress-test-nst

[13]F. Andreotti ; J. Oster ; G. D. Clifford, A Bayesian Filtering Framework for accurate extracting of the non-invasive FECG morphology Cambridge, MA 7-10 Sept.IEEE 2014

[14]Davide Anguita1, Alessandro Ghio, Luca Oneto, Xavier Parra2, and Jorge L. Reyes-Ortiz, Human Activity Recognition on Smartphones using a Multiclass Hardware-Friendly Support

Vector Machine, http://www.icephd.org/sites/default/files/IWAAL2012.pdf

[15]Seizure detection, seizure prediction, and closed-loop warning systems in epilepsy, Epilepsy \&

Behaviour 291-307, Elsevier (2014). 